\title{
EXPERIENCIA DE LAS PACIENTES FRENTE A CITOLOGÍA CÉRVICO-VAGINAL REPORTADA COMO ASCUS O LEI DE BAJO GRADO EN DOS INSTITUCIONES DE BOGOTÁ (COLOMBIA), 2014
}

\section{Patient experiences regarding ASCUS or low grade SIL cervicovaginal cytology results in two institutions in Bogotá (Colombia), 2014}

\author{
Solange Monsalve-Páez, $M D^{I}$; Diana Valderrama-Vega, $M D^{2}$; \\ Marcos Fidel Castillo-Zamora, MD ${ }^{3}$; Yahira Rossini Guzmán-Sabogal, MD $^{4}$; \\ Jairo Amaya-Guío, $M^{5}$
}

Recibido: abril 3/14 - Aceptado: febrero 10/15

\section{RESUMEN}

Objetivo: describir los conocimientos que tienen las pacientes acerca del uso de la citología, y las emociones y el comportamiento que genera en estas un resultado positivo en la citología vaginal con atipias escamosas de significado indeterminado (ASCUS) o lesión escamosa intraepitelial de bajo grado (LEI-BG).

Materiales y métodos: investigación cualitativa fenomenológica, que busca establecer las experiencias vividas por mujeres entre 18 y 69 años que consultaron por primera vez, por el hallazgo en la citología cérvico-vaginal de ASCUS o LEI-BG, a la unidad de patología cervical de dos instituciones de nivel medio de complejidad en Bogotá (Colombia), una institución pública y otra un centro de atención

1 Residente Ginecología y obstetricia, IV año, Universidad de La Sabana, Bogotá (Colombia).

2 Residente Ginecología y obstetricia, IV año, Universidad de La Sabana, Bogotá (Colombia). diana.valderrama@hotmail.es

3 Médico ginecólogo. Profesor Asistente, Universidad de La Sabana, Bogotá (Colombia).marcoscz@unisabana.edu.co

4 Médico psiquiatra. Profesor Asistente, Universidad de La Sabana, Bogotá (Colombia)

5 Médico ginecólogo. Profesor asociado, Departamento de Obstetricia y Ginecología, Universidad Nacional de Colombia, Bogotá (Colombia). ambulatorio privado. El tamaño de la muestra se determinó por saturación teórica y muestreo consecutivo. Se realizaron grupos focales y entrevistas, la información fue grabada y transcrita posteriormente, creando categorías para evaluar las experiencias: caracterización sociodemográfica, cognición, características afectivas y comportamiento. Se empleó el estilo de análisis mediante edición, presentado como material narrativo, a fin de ordenar la información recolectada para su síntesis e interpretación.

Resultados: se incluyeron 27 mujeres. El resultado anormal de la citología cérvico-vaginal despierta sentimientos de culpa, angustia y preocupación pues lo relacionan con el cáncer de cuello uterino, dado que no es claro para ellas que este es un examen de tamización. La principal conducta posterior a recibir el resultado de la citología fue priorizar la cita médica. Hay alteraciones en las relaciones de pareja basadas en la percepción de infidelidad secundaria a la infección de VPH.

Conclusiones: la toma de la citología cérvico-vaginal es considerada por las pacientes como importantísima y obligatoria en el marco de la necesidad de prevenir y tratar el cáncer de cuello uterino. 
Palabras clave: citología, investigación cualitativa, respuesta emocional.

\section{ABSTRACT}

Objective: To describe patient knowledge regarding the use of cytology, as well as the emotions and behaviours elicited by a positive vaginal cytology result of atypical squamous cells of undetermined significance (ASCUS) or low-grade squamous intraepithelial lesion (LG-SIL).

Materials and methods: Qualitative phenomenological research aimed at identifying experiences among women between 18 and 69 years of age presenting for the first time because of a cervicovaginal cytology finding of ASCUS or LG-SIL to the cervical pathology units of two intermediate complexity centres in Bogota, one of them a public institution and the other a private outpatient centre. The sample size was determined by theoretical saturation on the basis of a consecutive sampling. Focus groups and interviews were conducted, and the information was recorded and transcribed afterwards. Categories were created for experience assessment: socio-demographic and affective characteristics, cognition, and behaviour. Editing was used for a narrative analysis style in order to organize the information gathered for synthesis and interpretation purposes.

Results: Twenty-seven women were included. An abnormal cytology result gives rise to feelings of guilt and is associated with anxiety and fear of having cervical cancer, given that it is not clear to them that cervicovaginal cytology is a screening test. The main behaviour after receiving the cytology result was to give priority to the medical appointment. There are changes in the relationships with the partners due to perceived infidelity secondary to HPV infection.

Conclusions: Cervicovaginal cytology is is considered by patients of the greatest importance and mandatory given the need to prevent and treat cervical cancer.
Key words: Cytology, qualitative research, emotional response.

\section{INTRODUCCIÓN}

El cáncer de cérvix constituye un problema de salud pública alrededor del mundo y ocupa el tercer lugar en frecuencia entre las mujeres, siendo superado por el cáncer de seno y el cáncer de colon y recto. Se estima que al año se diagnostican en el mundo 500.000 nuevos casos, lo que representa más de 270.000 muertes atribuibles a esta condición; sin embargo, son los países con medianos y bajos ingresos aquellos que aportan en mayor medida a la carga de la enfermedad, al presentar mayores tasas de incidencia y de mortalidad cuando se comparan frente a los países desarrollados (1).

La citología cérvico-vaginal es una prueba de tamización que tiene por objeto identificar aquellas pacientes asintomáticas que cursan con una lesión premaligna, con el ánimo de proporcionar un tratamiento oportuno que disminuya la probabilidad de desarrollar una neoplasia (2). La expresión ASCUS corresponde a la sigla en inglés de células escamosas atípicas de significado no determinado, acuñada por la terminología Bethesda en 2001, que no necesariamente implica malignidad dado que, con gran frecuencia, su hallazgo se encuentra relacionado con un proceso netamente inflamatorio. Sin embargo, cuando se compara con la población general, las mujeres con ASCUS sí poseen un mayor riesgo de neoplasia por lo que requieren un seguimiento estricto y la realización de exámenes complementarios (2). Por otra parte, la lesión escamosa intraepitelial de bajo grado (LEI-BG) está asociada con infección por el virus del papiloma humano (VPH). La historia natural de las mujeres que presentan citologías con lesiones de bajo grado es similar a la de las mujeres que tienen citologías con ASCUS y VPH positivo; el riesgo de progresar a lesión de alto grado es de 3,8\%. Por ello se ha sugerido que estas mujeres deben tener un esquema de seguimiento similar (3). 
Una de las consecuencias más importantes de un resultado de citología anormal es la respuesta emocional de la paciente (4). Dentro de los principales factores que se han reconocido como influyentes en la respuesta emocional por parte de las pacientes frente a la citología vaginal, se encuentra la falta de conocimiento acerca del objetivo del examen y del significado del resultado (5). Por otra parte, muchas mujeres piensan que la citología cérvico-vaginal se realiza con el fin de detectar cáncer, lo que naturalmente conlleva que cualquier tipo de alteración reportada sea interpretada como el hallazgo de una patología maligna; además, se presenta miedo a sentir dolor y temor al rechazo en caso de recibir un resultado positivo (5). Asimismo, la manera con la que los profesionales de la salud informan los resultados también influye sobre la respuesta psicosocial de la paciente; de esta manera, mujeres a quienes se les entrega información educativa acerca del significado de la citología, presentan menores grados de ansiedad $(1,5)$.

Habitualmente, un resultado anormal o positivo en la citología vaginal genera sentimientos de culpa, rabia, confusión, ansiedad y depresión entre las pacientes $(6,7)$, lo que llega incluso a generar cambios en su imagen corporal con repercusiones negativas en la autoestima (8). Todo ello ligado a un profundo temor ante la posibilidad de requerir un tratamiento quirúrgico mutilante $(6,9)$.

Es poco lo que se conoce de las experiencias afectivas que experimentan las pacientes que reciben un resultado de citología anormal en nuestro medio. El conocimiento de la respuesta emocional frente a un resultado anormal en la citología vaginal, permitiría a los profesionales de la salud adoptar estrategias que incrementen la adherencia a los programas de diagnóstico y tratamiento de patología cervical, al tiempo que les brinda las herramientas necesarias para realizar una consejería encaminada a despejar las dudas y resolver los temores asociados al uso de esta herramienta diagnóstica.

El objetivo del presente estudio es describir los conocimientos que tienen las pacientes acerca del uso de la citología, y las emociones y el comportamiento que genera un resultado positivo en la citología vaginal (ASCUS o LEI-BG).

\section{MATERIALES Y MÉTODOS}

Diseño cualitativo fenomenológico de experiencias individuales vividas por pacientes con resultado anormal en la citología cérvico-vaginal (ASCUS o LEI-BG). Se incluyeron participantes de 18 a 69 años de edad que asistieron por primera vez al servicio de patología cervical para la realización de una colposcopia, en dos instituciones de salud de segundo nivel de complejidad de Bogotá: Hospital de Engativá, institución pública que atiende población del aseguramiento subsidiado, y Javesalud, entidad privada que atiende población adscrita al aseguramiento contributivo.

Se excluyeron pacientes con dificultades comunicativas o cognitivas, con diagnóstico confirmado de cáncer de cuello uterino o con enfermedad psiquiátrica. La muestra se realizó por conveniencia a partir de la identificación de casos consecutivos, hasta alcanzar el número necesario de participantes que permitió lograr la saturación teórica.

Procedimiento. Se desarrolló un borrador de entrevista y de grupo focal. Los instrumentos fueron sometidos a discusión durante un consenso de expertos (psiquiatra y ginecólogo) para luego ser validados mediante la realización de una prueba piloto con una paciente, con el ánimo de comprobar la claridad de las preguntas. El acercamiento a las pacientes se llevó a cabo antes de la consulta con el servicio de patología cervical (colposcopia), espacio durante el cual se dieron a conocer los objetivos del estudio y la forma en que podían participar para luego proceder a la aplicación de una entrevista semiestructurada en aquellas pacientes que aceptaron hacer parte del estudio.

Además, se realizaron dos grupos focales de ocho personas con sesión de dos horas de duración, con uno de los investigadores como moderador y otro como observador y relator. Para facilitar el análisis de información, la discusión se registró en 
grabadoras de voz y los archivos fueron transcritos textualmente para su posterior análisis mediante edición. Se extrajeron los datos con mayor significado para el objetivo del estudio y se identificaron aquellos códigos que permitieron la construcción de categorías, algunas emergentes y otras esperadas:

De forma general, se abordaron cuatro categorías: factores sociodemográficos, cognición, características afectivas y comportamiento. La caracterización sociodemográfica incluyó el estrato socioeconómico: los estratos 1 y 2 corresponden al nivel socioeconómico bajo, el 3 y el 4 al nivel medio, y el 5 y el 6 al nivel alto, el estado civil, la escolaridad y el conocimiento sobre la cobertura y acceso a la citología.

Los datos fueron analizados de forma independiente por dos de los autores. Se realizó la triangulación de la información obtenida. Quienes realizaron el análisis de la información compararon los resultados y, en caso de discrepancia, esta fue resuelta mediante consenso. Un investigador externo al proyecto y con conocimiento del tema, examinó la validez de las conclusiones.

Aspectos éticos. Se pidió el consentimiento informado; para ello, los participantes otorgaron autorización escrita para grabar las entrevistas y se protegió la confidencialidad de la información. El comité de ética de la Universidad de La Sabana aprobó el estudio.

\section{RESULTADOS}

Se exponen los resultados obtenidos, a partir de una muestra de 27 mujeres entrevistadas entre julio y diciembre de 2013.

Caracterización sociodemográfica. Con respecto al estado civil, predominaron las pacientes solteras, viudas o separadas (62\%), el $38 \%$ estaban casadas o en unión libre. En cuanto a la escolaridad, el 66\% de las pacientes había cursado primaria o secundaria, con una mayor frecuencia en la institución pública, y en el nivel universitario o técnico el 34\%, con un mayor peso del grupo entrevistado en la institución privada. En cuanto al estrato socioeconómico, el 19\% correspondió a los estratos 1 y 4 respectivamente, el $22 \%$ al estrato 2, el $25 \%$ al estrato 3 y el $15 \%$ a los estratos 5 y 6 . Por otra parte, se tiene la percepción de que en los niveles más bajos se utiliza menos el examen aunque está cubierto por todos los regímenes de aseguramiento. Es importante resaltar que algunas mujeres no saben que en el sistema de seguridad social en Colombia dicho examen es gratuito.

Cognición. Se pudieron percibir tres subcategorías que fueron comunes entre las participantes: la terminología, la necesidad y el tiempo para la realización del examen. En cuanto a la terminología para referirse a la citología cérvico-vaginal, las mujeres comparten con el campo de la medicina formal la denominación de "examen” para definir la citología cérvico-vaginal. Las mujeres relacionan la citología con el cáncer y con las infecciones vaginales. En cuanto a la necesidad de la citología cérvico-vaginal, todas las mujeres, en ambos centros de salud, la consideran como "importantísima" y "obligatoria" en el marco de la necesidad para "tratar" el cáncer de cuello uterino. No tienen claro el esquema de screening de la citología, esto implica una confusión importante para un examen de tamización. Respecto al tiempo de toma consideran que debe ser máximo con una frecuencia anual (tabla 1).

Categoría características afectivas. Para la mayoría de las mujeres entrevistadas el significado de la citología cérvico-vaginal es tener una infección de transmisión sexual con el VPH; sin embargo, ellas no tienen claro lo que es el VPH. Identifican los resultados de su citología con algo malo y que es debido a su pareja sexual. Algunas participantes del Hospital de Engativá consideran que dichas alteraciones pueden ser desarrolladas por falta de higiene. El resultado anormal de la citología cérvico-vaginal despierta sentimientos de angustia y preocupación pues lo relacionan con el cáncer de cuello uterino, dado que no es claro para ellas que este es un examen de tamización. Algunas refieren un sentimiento de culpa acerca de sus conductas sexuales en el pasado. La mayoría de pacientes concuerdan en que recibieron "una llamada" por parte del personal de enfermería, 


\begin{tabular}{|c|c|}
\hline Categoría cognición & Código identificado \\
\hline \multirow[t]{2}{*}{$\begin{array}{l}\text { Terminología para referirse a citología } \\
\text { cérvico-vaginal }\end{array}$} & $\begin{array}{l}\text { "Es una revisión anual para saber cómo es el estado del cuello } \\
\text { uterino" }\end{array}$ \\
\hline & $\begin{array}{l}\text { "La verdad que sirve para detectar el cáncer de cuello uterino } \\
\text { y de alguna enfermedad" }\end{array}$ \\
\hline \multirow[t]{2}{*}{$\begin{array}{l}\text { Necesidad de realización de la citología } \\
\text { cérvico-vaginal }\end{array}$} & $\begin{array}{l}\text { "Claro, porque eso nos previene muchas cosas, antes de } \\
\text { cualquier enfermedad y todo" }\end{array}$ \\
\hline & $\begin{array}{l}\text { "Sí, demasiado importante porque detecta enfermedades } \\
\text { como el papiloma y el cáncer" }\end{array}$ \\
\hline \multirow[t]{2}{*}{ Tiempo de realización de la citología } & “Cada año" \\
\hline & "En algunos casos cada año y cada 6 meses" \\
\hline
\end{tabular}

y solo una paciente refirió haber recibido dicha información “por Internet”. En la mayoría de casos recibieron una adecuada información, con amabilidad y privacidad, resaltando el hecho de llamarlas como una actitud de interés del sistema de salud hacia ellas. Sin embargo, la mitad de las pacientes resaltaron que no hubo claridad en la información suministrada; en general, estas concuerdan en reforzar la claridad de los conceptos por parte del personal de salud -algo concreto, sin terminología médica- (tabla 2).

Categoría comportamiento. La principal conducta posterior a recibir el resultado de la citología fue priorizar la cita médica; al mismo tiempo, muchas buscaron por Internet, evidenciando que para los estratos bajos la información suministrada fue más confusa que para los estratos altos, con mayor nivel de conocimiento, sin ser clara completamente. Otras mujeres consideraron avisar a su pareja, lo que de cierta forma les dio tranquilidad cuando esta les reforzaba la idea de no engaño. La mayoría de mujeres concuerdan en cierta desconfianza hacia su pareja, por su pasado o presente (tabla 3).

\section{DISCUSIÓN}

En este estudio se observó que las mujeres no identifican la citología como un examen de inspección, sino que la relacionan con el cáncer y con las infecciones de transmisión sexual. Consideran la toma de la citología cérvico-vaginal como "importantísima" y "obligatoria" en el marco de la necesidad para “tratar" el cáncer de cuello uterino. Las mujeres entrevistadas relacionan el resultado anormal de la citología cérvico-vaginal con sentimientos de angustia, miedo y preocupación por tener alguna enfermedad o cáncer de cuello uterino. Algunas, con un sentimiento de culpa. Refieren que recibieron una adecuada información con amabilidad y privacidad; sin embargo, resaltaron que no hubo claridad en la información suministrada. Ante el resultado positivo la conducta más frecuente fue priorizar la cita médica; en algunos casos, avisar a la pareja. Hay desconocimiento sobre el acceso a esta prueba en el sistema de seguridad social en salud.

Nuestros hallazgos concuerdan con los resultados documentados en otros estudios. A pesar de una primera reacción de desesperanza en las participantes, surgió un proceso posterior de toma de conciencia y afrontamiento en las mujeres que las llevó a continuar con su ejercicio diagnóstico, como fue asistir a la realización de la colposcopia. La esencia del afrontamiento basado en la solución de problemas tiene en cuenta la espiritualidad con el fin de fortalecer las esperanzas del paciente, señala que "es relevante impartir conocimientos acerca de la enfermedad, las terapias utilizadas, con información clara y veraz" $(10,11)$. 


\begin{tabular}{|c|c|}
\hline Categoría: Características afectivas & Código identificado \\
\hline \multirow[t]{3}{*}{$\begin{array}{l}\text { Ambiente de información del resultado } \\
\text { de la citología }\end{array}$} & $\begin{array}{l}\text { "Una enfermera me llamó que habían algunas anomalías, } \\
\text { para hacerme un nuevo procedimiento" }\end{array}$ \\
\hline & $\begin{array}{l}\text { "Me llegó un correo, la EPS* me mandó el examen, yo lo } \\
\text { revisé y me di cuenta que estaba mal, ahí procedí a sacar una } \\
\text { cita al médico" }\end{array}$ \\
\hline & "Fue privado y adecuado" \\
\hline \multirow{3}{*}{$\begin{array}{l}\text { Opinión acerca de la información } \\
\text { suministrada }\end{array}$} & "Me pareció una forma adecuada, me aclararon el resultado" \\
\hline & $\begin{array}{l}\text { "No me informaron bien del examen, no entendía nada, no } \\
\text { hubo claridad en esa parte de información" }\end{array}$ \\
\hline & $\begin{array}{l}\text { "Es importante dar información pero no por medio de un } \\
\text { correo, es mejor que lo llamen" }\end{array}$ \\
\hline \multirow[t]{3}{*}{$\begin{array}{l}\text { Significado personal del resultado anormal } \\
\text { de la citología }\end{array}$} & $\begin{array}{l}\text { "Uno no sabe qué tiene, es preocupante tener cáncer o algo y } \\
\text { no estar informado" }\end{array}$ \\
\hline & $\begin{array}{l}\text { "Yo he tratado de ser organizada, limpia, responsable, uno } \\
\text { con mi vida sexual, dos con mi higiene personal" }\end{array}$ \\
\hline & $\begin{array}{l}\text { "Que no es nada bueno, por lo que le dicen a uno del cáncer } \\
\text { del cuello uterino" }\end{array}$ \\
\hline \multirow[t]{3}{*}{$\begin{array}{l}\text { Sentimiento acerca del resultado } \\
\text { de la citología }\end{array}$} & $\begin{array}{l}\text { "Sentí miedo, me da miedo tener alguna enfermedad o un } \\
\text { cáncer o algo así" }\end{array}$ \\
\hline & "Preocupación de tener algo grave" \\
\hline & $\begin{array}{l}\text { "Mi pareja es estable, pero uno no sabe el pasado de la } \\
\text { persona, uno se da cuenta mucho tiempo después" }\end{array}$ \\
\hline \multirow[t]{2}{*}{ Sugerencias } & $\begin{array}{l}\text { "Que sean más claros con la información, no importa saber } \\
\text { de una, pero saber" }\end{array}$ \\
\hline & $\begin{array}{l}\text { "Es importante dar información pero no por medio de un } \\
\text { correo" }\end{array}$ \\
\hline
\end{tabular}

* EPS: término utilizado para las empresas aseguradoras del régimen contributivo.

Brindar información clara y precisa conlleva indudables beneficios: a) contribuye de manera significativa a la disminución de estados emocionales alterados (presencia de ansiedad, estrés y depresión); b) propicia apego y respuesta al tratamiento, y c) disminuye demandas por mala práctica (12, 13). Tal como Peiró et al. señalan, comunicar malas noticias es una tarea compleja; más aún cuando la enfermedad por comunicar incluye la palabra cáncer, pues el término posee connotaciones culturales negativas; como se pudo observar en nuestro estudio, esto puede llevar a desconfianza, alterando la vida de pareja (14).

Desde esta perspectiva, es relevante el desarrollo de un grupo multidisciplinario que incluya al ginecólogo, psicólogo o psiquiatra para la realización de dicha tamización, prestando un servicio integral, con alto nivel humano y científico, usando herramientas 


\begin{tabular}{|l|l|}
\hline $\begin{array}{l}\text { Tabla } 3 . \\
\text { Descripción de los códigos que corresponden a la categoría comportamiento respecto a los } \\
\text { resultados de citología ASCUS y LEI-BG en pacientes en dos instituciones de Bogotá (Colombia) }\end{array}$ \\
\hline Categoría comportamiento & Código identificado \\
\hline Conducta posterior al resultado de la citología & "Busqué en Internet y me confundí más" \\
\hline comportamiento posterior al resultado & "No dude en ir al médico" \\
\hline "Las relaciones sexuales han disminuido, digamos para no \\
contaminarlo, por higiene, para no afectarlo a él” \\
"Pues me produce un poco de desconfianza con mi pareja, \\
porque me da miedo tener una enfermedad por culpa de él; \\
no sé, en eso pienso"
\end{tabular}

de acuerdo con la caracterización de la población que se va a intervenir, a fin de facilitar la comunicación no solo del diagnóstico de cáncer, sino de toda la historia natural del mismo, incluyendo las condiciones de riesgo para su desarrollo $(15,16)$. Para este caso en particular (comunicación de citología como anormal), es recomendable que el uso de técnicas de comunicación efectiva no se restrinja a los médicos especialistas $(11,17)$.

\section{CONCLUSIONES}

El resultado anormal de la citología cérvico-vaginal se relaciona con angustia, miedo y preocupación por tener alguna enfermedad o cáncer de cuello uterino. Algunas veces con un sentimiento de culpa por sus conductas sexuales en el pasado. La principal conducta posterior a recibir el resultado de la citología fue priorizar la cita médica.

El personal de salud que realiza la citología debe ser claro en el objetivo de este examen como de tamización y no de diagnóstico, informando adecuadamente las conductas que se deben seguir con el fin de disminuir los posibles efectos psicosociales a corto y largo plazo.

\section{REFERENCIAS}

1. Ferlay J, Soerjomataram I, Ervik M, Dikshit R, Eser S, Mathers C, et al. Cancer Incidence and Mortality Worldwide: IARC. Globocan. 2012; v1.0.
2. Instituto Nacional de Cancerología. Guía de Práctica Clínica. Recomendaciones para el tratamiento de las pacientes con citología reportada con células escamosas atípicas de significado indeterminado (ASC-US) en Colombia; 2007. [Visitado: 2014 Feb 14]. Disponible en: http://www.nacer.udea.edu.co/ pdf/jornadas/2ascus2.pdf

3. Stewart Massad L, Einstein MH, Huh WK, Katki HA, Kinney WK, Schiffman M, et al. Update Consensus Guidelines for the management of Abnormal Cervical Cancer Screening Test and Cancer Precursors. American Society for Colposcopy and Cervical Pathology Journal of Lower Genital Tract Disease. 2013;17:S1-S27.

4. Monsonego J, Cortes J, da Silva DP, Jorge AF, Klein P. Psychological impact, support and information needs for women with an abnormal Pap smear: comparative results of a questionnaire in three European countries. BMC Womens Health. 2011;11:18.

5. Herzog T, Wright J. The impact of cervical cancer on quality of life-the components and means for management. Gynecol Oncol. 2007;107:572-7.

6. Petticrew MP, Sowden AJ, Lister-Sharp D, Wright K. False-negative result in screening programmes: systematic review of impact and implications. Health Technol Assess. 2000;4:1-120.

7. Bonelli L, Branca M, Ferreri M, Barizzone D, Rossi E, Cedri S, et al. Attitude of women towards early cancer detection and estimation of the compliance 
to a screening program for cervix and breast cancer. Cancer Detect Prev. 1996;20:342-52.

8. Hellsten C, Sjöström K, Lindqvist PG. A longitudinal 2-year follow-up of quality of life in women refer for colposcopy after an abnormal cervical smear. Eur J Obstet Gynecol Reprod Biol. 2009;147:221-5.

9. Campbell H, MacDonald S, McKiernan M. Promotion of cervical screening uptake by health visitor follow-up of women who repeatedly failed. J Public Health Med. 1996;18:94-7.

10. Escobar J. Grupos Focales una guía conceptual y metodológica. Cuadernos Hispanoamericanos de Psicología. 2010;9:57-6.

11. Larios FE, Robles R, Jiménez LM, Suárez AE, del Toro A, Danieri A. Información y soporte emocional a las mujeres con resultado anormal en Papanicolaou: una guía de comunicación. Psicooncología. 2009;6:155-66.

12. Zeisler H, Mayerhofer K, Joura EA, Sator M, Kainz C. Psychological burden of women with mild cervical intraepithelial neoplasia. Oncol Rep. 1997;4:1063-5.

13. Simon MA, Cofta-Woerpel L, Randhawa V, John P, Makoul G, Spring B. Using the word 'cancer' in communication about an abnormal Pap test: finding common ground with patient-provider communication. Patient Educ Couns. 2010;81:106-12.

14. Peiró G, Corbellas C, Camps C, Sánchez PT, Peiró G, Godes M, et al. Estudio descriptivo sobre el proceso de comunicar el diagnóstico y el pronóstico en oncología. Psicooncología. 2006;3:9-17.

15. Fylan F. Screening for cervical cancer: a review of women's attitudes, knowledge, and behaviour. Br J Gen Pract. 1998;48;1509-14.

16. Maissi E, Marteau ET, Hankins M, Moss S, Legood R, Gray A. Psychological impact of human papilloma virus testing in women with borderline or mildly dyskaryotic cervical smear test results: cross sectional questionnaire study. BMJ. 2004;328:1293.

17. McCaffery K, Waller J, Nazroo J, Wardle J. Social and psychological impact of HPV testing in cervical screening: a qualitative study. Sex Transm Infect. 2006;82:169-74. 